\title{
The Sniffin' Sticks Odor Discrimination Memory Test: A Rapid, Easy-to-Use, Reusable Procedure for Testing Olfactory Memory
}

\author{
Gerold Besser, MD' ${ }^{(D}$, Leandra Jobs, MD', David Tianxiang Liu, MD', \\ Christian A. Mueller, MD', and Bertold Renner, MD ${ }^{2}$
}

\begin{abstract}
Objectives: Neurodegenerative diseases can alter odor memory in addition to inducing quantitative impairment, and olfactory memory can be tested using the validated olfactory memory test with encapsulated odors (Odor Discrimination Memory Test [ODMT]). The aim of this study was to investigate the new Sniffin' Sticks ODMT (SSODMT), which is comparable with the ODMT.

Methods: The SSODMT was administered to 48 subjects ( 30 women, 18 men; mean age, $34.6 \pm 16.3$ years). Olfactory function was assessed using the 16-item Sniffin' Sticks Identification Test. The ODMT was administered to a subgroup of 10 subjects to assess the comparability of the SSODMT and the ODMT. Test-retest reliability was studied in another 20 subjects ( 10 women, 10 men; mean age, $32.9 \pm 16.4$ years).

Results: The mean SSODMT score was $10.7 \pm 1.8$ (range, 5-I2). SSODMT test scores were significantly correlated with Sniffin' Sticks Identification Test scores $\left(r_{67}=0.66, P<.00 I\right)$ and were significantly negatively correlated with subject age $\left(r_{48}=-0.70, P<.00 \mathrm{I}\right)$. SSODMT and ODMT scores were highly and significantly correlated, indicating that the test results were comparable $\left(r_{10}=0.75, P<.05\right)$. Test-retest analysis revealed a significant correlation $\left(r_{20}=0.68, P<.00 I\right)$.

Conclusions: These results demonstrate the usefulness of the new SSODMT and its comparability with the ODMT. The SSODMT is an easy, rapid, and low-cost tool that is suitable for routine use in evaluating odor memory.
\end{abstract}

\section{Keywords}

odor memory, olfaction, neurodegenerative, reusability, smell

\section{Introduction}

Assessment of olfactory function is an important diagnostic tool in a variety of diseases. ${ }^{1}$ Olfactory dysfunction (OD) can be due to conditions treated within the specialty of otorhinolaryngology, including sinonasal-, infectious disease-, and allergy-based conditions. In addition, numerous medical conditions and some neurodegenerative diseases, such as Alzheimer's disease and Parkinson's disease (PD), can lead to OD. Indeed, OD is an important PD marker that affects more than $90 \%$ of patients with $\mathrm{PD},{ }^{2}$ and the presence of normal olfactory function raises the question of whether PD is the correct diagnosis. ${ }^{3}$ Patients with neurodegenerative diseases are often unaware that they have OD, as are many patients with OD in general. ${ }^{4}$ In addition to quantitative impairment, patients with neurodegenerative diseases may also have altered odor memory. ${ }^{5}$ Validated odor memory tests are very valuable in this context.
A number of smell tests have been validated for the quantitative measurement of the sense of smell in humans. These tests can be used both to diagnose OD and to monitor therapeutic success. ${ }^{6-10}$ The University of Pennsylvania Smell Identification Test is one such test that is used widely. This test for OD, which can be self-administered, uses up to 40 encapsulated odors in a forced-choice paradigm. ${ }^{10}$ These encapsulated odors have also been validated for testing

\footnotetext{
'Department of Otorhinolaryngology-Head and Neck Surgery, Medical University of Vienna, Vienna, Austria

${ }^{2}$ Institute of Experimental and Clinical Pharmacology and Toxicology, Erlangen, Erlangen, Germany

Corresponding Author:

Christian A. Mueller, MD, Department of Otorhinolaryngology-Head and Neck Surgery, Medical University of Vienna, Währinger Gürtel I820, Vienna, 1090, Austria

Email: christian.a.mueller@meduniwien.ac.at
} 
olfactory memory in the Odor Discrimination Memory Test (ODMT) and are commercially available. ${ }^{11,12}$

The Sniffin' Sticks test battery is also very commonly used for research (Burghart GmbH, Wedel, Germany). This battery tests different "olfactory dimensions" by testing for threshold, discrimination, and identification using hygienic and reusable odorant "pens." ${ }^{.13}$ Summed threshold, discrimination, and identification scores can be compared with normative data to differentiate between normosmia, hyposmia, and anosmia. ${ }^{14}$ Zucco $^{15}$ reported an odor recognition test that uses the 16-item Sniffin' Sticks battery to test odor identification. In this test, a subject is presented 1 odor and 10 seconds later must recall the target odor out of 3 odors. Croy et $\mathrm{al}^{16}$ proposed an odor memory test that uses Sniffin' Sticks. ${ }^{16}$ In this test, 8 target odors are presented once in the course of an initial memorizing phase. Shortly after presentation and during the same session, the subject is presented with 16 odors and is asked to recognize the initial 8 odors. These procedures differ from the ODMT, which provides just 4 target odors and has separate recognition testing sessions for each odor after preset time periods $(10,30$, and 60 seconds), with the remaining 3 odors as descriptors. ${ }^{12}$ All of these test strategies use a delayed matching-to-sample paradigm that is used to test the memories of animals and humans. ${ }^{17}$

The aim of the present study was to assess an odor memory test that is comparable to the ODMT and that uses reusable Sniffin' Sticks.

\section{Methods}

Participants were recruited using invitational notices that were displayed on the campus of the Medical University of Vienna. The study was carried out according to the guidelines of the Declaration of Helsinki on Biomedical Research Involving Human Subjects and was approved by the local ethics committee (EK-No. 1605/2014). All subjects provided written informed consent prior to participation.

\section{Experimental Subjects}

The study included 48 healthy subjects, 30 women and 18 men, with a mean age of $34.6 \pm 16.3$ years (range, 18-87 years). At the beginning of the study, each participant underwent a clinical examination and completed a questionnaire (including subjective self-assessment of smell function). Prior to the smell tests, participants rated their subjective olfactory function (SOF) on a 100 -mm linear scale that ranged from 0 (no sense of smell) to 100 (excellent sense of smell). The mean SOF score of the participants was $59.1 \pm 20.4$ points. Subjective nasal ventilation was assessed separately for each nasal side on a numeric scale that ranged from 0 (very bad) to 7 (excellent). The mean subjective nasal ventilation scores were $4.1 \pm 1.3$ for the left side and $4.2 \pm 1.3$ for the right side. Only 9 participants indicated that their subjective nasal ventilation differed on the left and right sides, but the difference was just 1 point in all 9 subjects, except for 1 subject who had a 3-point difference because of a deviated septum. Of the 48 participants, 17 (35.4\%) were smokers. Prior to smell testing, cognitive function was assessed using 3 tests, the Mini Mental State Examination (MMSE), the Wortschatztest (WST), and the Advanced Progressive Matrices (APM). Subjects did not eat, drink, or smoke for at least 1 hour prior to the tests.

\section{Cognitive Tests}

The MMSE, a short test of overall cognitive function, consists of 11 tasks related to orientation, memory, attention, calculation, and verbal and motor abilities. ${ }^{18}$ The maximum score is 30 ; scores of 24 to 30 indicate normal cognitive function, scores of 18 to 23 indicates mild cognitive dysfunction, and scores of 10 to 17 indicate severe cognitive dysfunction. The mean MMSE score was $29.9 \pm 0.4$, and none of the participants had scores less than 24 , indicating normal cognitive function.

The WST, a test of verbal intelligence, consists of 42 items of increasing difficulty. ${ }^{19}$ Each item is a list of 5 words that are not real and 1 that is real. The subject must identify the real word, and the maximum score is 42 . The WST takes about 5 minutes and can be used several times with little to no learning effect. ${ }^{19}$ The mean WST score was $34.2 \pm 3.0$ (range, 26-39), which was comparable with normative data.

Of the 48 participants, 30 completed the APM, which is a test of nonverbal intelligence and logic. ${ }^{20}$ They used Set I of the APM, which has 12 visually presented items with 8 response choices. All 12 items had to be completed, with no time limit. The mean APM test score was $10.6 \pm 1.5$ (range, $7-12$ ), which was comparable with normative data. ${ }^{21}$

\section{Odor Memory Test}

The new Sniffin' Sticks ODMT (SSODMT) uses 4 odors that are used in the validated Sniffin' Sticks Identification Test (SSIT): rose, peppermint, banana, and shoe leather. These odors have shown a high identification rate in previous investigations ${ }^{22}$ and were selected because they overlap with those used in the ODMT, which uses rose, peppermint, banana, and peanut. Peanut odor is not part of the 16-item SSIT.

The SSODMT is a single-target, 4-alternative, forcedchoice procedure without verbal cues. After the presentation of 1 odor stick (the target odor), the blinded subject must memorize the odor with minimal verbal cues for a certain time period $(10,30$, or 60 seconds). During this time period, the subject must start counting backward from 280 by subtracting 3 . After the time period, all 4 odors are presented in a randomized manner, and the subject has to 
Table I. Administration Strategy for the Sniffin' Sticks Odor Discrimination Memory Test. ${ }^{\text {a }}$

\begin{tabular}{lcc}
\hline Target Odor & Period (s) & Response Set Choices \\
\hline I. Rose & 10 & Banana/shoe leather/rose/peppermint \\
2. Peppermint & 30 & Rose/peppermint/banana/shoe leather \\
3. Shoe leather & 30 & Banana/rose/peppermint/shoe leather \\
4. Banana & 60 & Banana/shoe leather/rose/peppermint \\
5. Peppermint & 10 & Peppermint/banana/rose/shoe leather \\
6. Rose & 60 & Banana/rose/shoe leather/peppermint \\
7. Shoe leather & 60 & Peppermint/banana/shoe leather/rose \\
8. Banana & 30 & Shoe leather/peppermint/banana/rose \\
9. Peppermint & 60 & Rose/banana/shoe leather/peppermint \\
10. Rose & 30 & Rose/peppermint/banana/shoe leather \\
II. Banana & 10 & Shoe leather/rose/peppermint/banana \\
12. Shoe leather & 10 & Banana/shoe leather/peppermint/rose \\
\hline
\end{tabular}

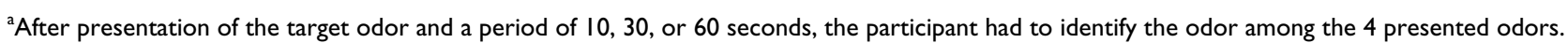

indicate the memorized odor. Each of the 4 target odors is presented a total of 3 times, once after 10 seconds, once after 30 seconds, and once after 60 seconds (Table 1). Each correct answer for the 12 trials is scored as 1 point, for a maximum score of 12 points.

In this study, each subject had to read the following instructions prior to taking the SSODMT:

You will smell an odor stick, and you must memorize its odor. There is a total of 12 odor sticks. After smelling the stick, please count backwards out loud from 280 in steps of 3 until the investigator stops you and presents 4 odors sticks consecutively. One of the odor sticks is the odor you had to memorize. Please indicate this odor. If you are unsure, you must guess.

After the SSODMT, 32 of the 48 subjects were asked to describe the strategies they used to memorize the target odors. Multiple answers were possible: "the odor itself," "a visual cue," "a verbal cue," "a color cue," or "an unspecified cue." Then the participants had to label the 4 odorants. A subgroup of 10 subjects was also administered the ODMT (using encapsulated odors) to determine the comparability of the SSODMT and the ODMT.

\section{Quantitative Olfactory Testing}

After the SSODMT was administered, the complete 16-item SSIT was administered. This test screens for normosmia, hyposmia, or anosmia. ${ }^{9}$ One subject scored 6 points on the identification test, suggesting OD. One subject scored 10 and 1 scored 12, indicating hyposmia, but all others scored within the normal range of normosmia (mean score, $13.9 \pm 1.6$ ).

\section{Test-Retest Reliability}

Another 20 subjects, 10 women and 10 men, with a mean age of $32.9 \pm 16.4$ years (range, $18-75$ years) were tested twice with a short break in between. The break was used for the MMSE, where the mean MMSE score was $29.5 \pm 0.8$ and hence comparable with the initial healthy cohort of 48 subjects. Also, the SOF ratings were comparable with the initial group, with a mean score of $62.0 \pm 21.1$ points. Four subjects within this group were patients with OD due to idiopathic $(\mathrm{n}=2)$ or postinfectious olfactory loss $(\mathrm{n}=2)$.

\section{Statistical Analysis}

SPSS 23.0 (IBM Corporation, Chicago, Illinois, USA) was used for statistical analysis. To compare scores in an explorative manner, we used unpaired 2-sample $t$ tests without adjustment for multiple comparisons. The normality of data was tested using the Shapiro-Wilk test. Correlational analyses were performed using the Pearson correlation coefficient. Bonferroni tests or Tukey's $b$ was used for post hoc comparisons as appropriate. The $\alpha$ level was set at 0.05 . Prism 7.0 (GraphPad Software, La Jolla, California, USA) was used to visualize data.

\section{Results}

Table 2 shows the subjects' scores according to sex. The sex distribution of the participants was uneven, but only SOF scores showed sex-specific differences. The SOF score was significantly higher in female subjects, but overall, sex did not correlate significantly with SSODMT and $\operatorname{SSIT}(P>.05)$.

The mean SSODMT score was $10.7 \pm 1.8$ (range, 5-12). The maximum score was 3 points for each odor stick, and the 4 different odor sticks showed similar mean scores: rose, $2.6 \pm 0.6$; peppermint, $2.7 \pm 0.5$; banana, $2.6 \pm 0.8$; and shoe leather, $2.7 \pm 0.5$. The maximum score was 4 points for each time period, and the mean scores for the 3 different time periods were as follows: 10 -s period, $2.7 \pm 0.5 ; 30$-s 
Table 2. Test Scores According to Sex.

\begin{tabular}{lcrrrr}
\hline & \multicolumn{2}{c}{ Female } & & \multicolumn{2}{c}{ Male } \\
\cline { 2 - 3 } \cline { 6 - 6 } Variable & Mean & SD & & Mean & SD \\
\hline Age & $33.3(n=30)$ & 18.4 & & $35.8(n=18)$ & 14.6 \\
SSIT & $13.8(n=30)$ & 1.7 & & $14.0(n=17)$ & 1.5 \\
SSODMT & $10.8(n=30)$ & 2.0 & & $10.4(n=18)$ & 1.5 \\
APM & $10.1(n=18)$ & 1.6 & & $11.3(n=12)$ & 1.0 \\
WST & $33.6(n=30)$ & 3.2 & & $35.2(n=18)$ & 2.5 \\
SOF & $65.5^{\mathrm{a}}(\mathrm{n}=30)$ & 17.4 & & $48.4^{\mathrm{a}}(\mathrm{n}=18)$ & 4.9 \\
\hline
\end{tabular}

Abbreviations: APM, Advanced Progressive Matrices; SOF, subjective olfactory function; SSIT, Sniffin' Sticks Identification Test; SSODMT, Sniffin' Sticks Odor Memory Discrimination Test; WST, Wortschatztest. ${ }^{\mathrm{a}} \mathrm{P}<.05$.

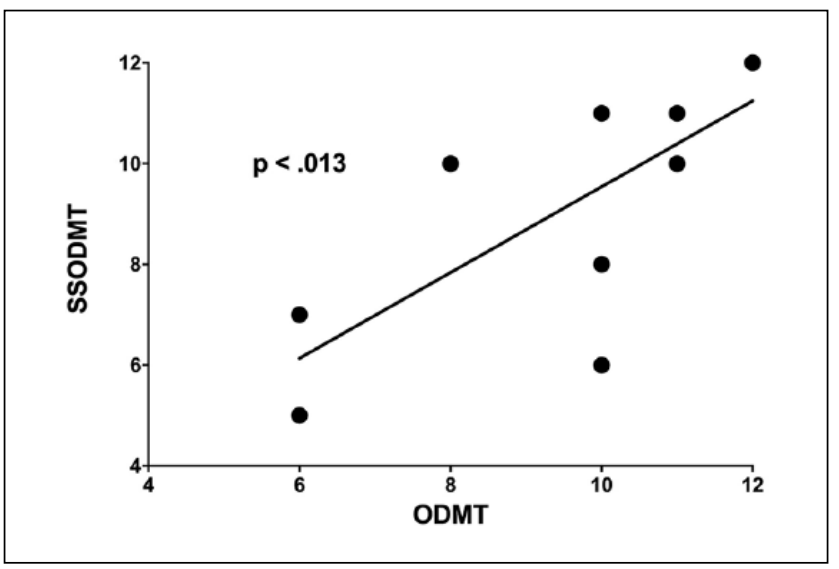

Figure I. Correlation of the Odor Discrimination Memory Test (ODMT) using encapsulated odors and the Sniffin' Sticks ODMT (SSODMT) $\left(r_{10}=0.75, P<.013\right)$.

period, $3.6 \pm 0.7$; and 60 -s period, $3.4 \pm 0.8$ points. The mean score for the 10 -s period was significantly lower than that for the 30 -s and 60 -s time periods $(P<.001)$.

The SSODMT scores showed significant negative correlations with subjects' age $\left(r_{48}=-0.70, P<.001\right)$ and WST scores $\left(r_{48}=-0.29, P<.05\right)$ and significant positive correlations with APM scores $\left(r_{30}=0.40, P<.05\right)$. In addition, the APM results showed significant negative correlations with increased age $\left(r_{30}=-0.47, P<.05\right)$, indicating worse APM results in older subjects. Older participants scored significantly better on the WST $\left(r_{48}=0.30, P<.05\right)$. Age also influenced the SSIT score, with older subjects scoring worse on the SSIT $(P<.05)$. MMSE scores did not show significant correlations with SSODMT scores $(P>.05)$.

The mean score for the validated ODMT in 10 subjects was $9.6 \pm 2.2$ (range, 6-12); this showed significant correlation with the SSODMT score $\left(r_{10}=0.75, P<.05\right)$ (Figure 1).

The strategies for memorization $(\mathrm{n}=32$, multiple answers) were as follows: "odor itself" $(\mathrm{n}=19)$, "visual cue" $(\mathrm{n}=16)$, "verbal cue" $(\mathrm{n}=23)$, "color cue" $(\mathrm{n}=4)$, and "unspecified cue" $(\mathrm{n}=11)$. Subjects using verbal cues did not score significantly higher on the WST, and those using visual cues did not score significantly higher on the APM $(P>.05)$. Notably, 7 subjects reported using a single cue, 14 reported using 2 cues, and 11 subjects used 3 or 4 memorizing strategies. As for free labeling of the odors, of the 31 participants, $48.4 \%$ correctly identified rose, $51.6 \%$ peppermint, $67.7 \%$ banana, and $3.2 \%$ shoe leather.

In a group of 20 subjects, including 4 patients with OD, the mean SSODMT score was $9.7 \pm 2.5$ (range, 3-12) for the first session and $9.4 \pm 2.3$ (range, 2-12) for the second session. Test-retest analysis revealed a significant correlation $\left(r_{20}=0.68, P<.001\right)$.

When gathering all subjects, the SSODMT scores correlated significantly with the SSIT scores $\left(r_{67}=0.66, P<.001\right)$.

\section{Discussion}

The main finding in the present investigation was that the SSODMT was a rapid and easy-to-use tool for testing olfactory memory. The SSODMT involves minimal and reusable resources, is relatively inexpensive, and is suitable for use as a routine tool in doctors' offices, regardless of their specialties.

Odor memory plays an integral role in cognitive function, and it can be used to judge cognitive function as well as olfactory function in a way that differs from odor threshold measurements and odor identification. The incidence of OD in neurodegenerative diseases, such as Alzheimer's disease and $\mathrm{PD}$, underlines the need to conduct odor memory tests to allow broader diagnostic possibilities.

We chose to test selected items of the SSIT in a way that was similar to the ODMT as published by Doty et al, ${ }^{22}$ because of the ODMT's validated and easy-to-use protocol. With the SSODMT, olfactory memory was tested using 3 defined time periods, ranging from 10 to 60 seconds, with a distracting task (counting) in between the intervals to minimize the use of explicit verbal memorizing strategies. Even though the group of subjects who were tested with both the ODMT and the SSODMT was rather small $(\mathrm{n}=10)$, the data indicate that the 2 are comparable: both test odor memory using either encapsulated odors (the ODMT) or "odorfilled" pens or Sniffin' Sticks (the SSODMT). Notably, our data for the SSODMT show similar test-retest reliability coefficients as Doty et $\mathrm{a}^{22}$ found using the ODMT.

The 4 odors chosen for the SSODMT were identified correctly by a high percentage of participants during olfactory testing in a previous study. ${ }^{23}$ The SSOMDT is a nonverbal test, but at the end of the test, we asked the participants to name the presented odors. Similar to how they recognize faces, humans also tend to be good at recalling odors; however, labeling or describing odors seems to be difficult. ${ }^{24}$ Uncued odor identification levels are generally quite low (seldom higher than 50\%). ${ }^{25}$ Unpublished data from our 
group showed uncued odor identification levels as low as $26 \%$ in healthy subjects when being confronted with 24 odors out of the Le Nez du Vin odor set $(n=44) .{ }^{26}$ However, people often experience the so-called tip-of-the-nose phenomenon, meaning that a specific odor may seem familiar, but one cannot recall its name. ${ }^{27}$ When the odor is named, it is immediately recognized. In our study, shoe leather was correctly identified considerably fewer times than the other odors. This may be because leather has lower hedonic character (unpleasant), and its odor is not connected to pleasant things, in contrast to the odors of banana, peppermint, and flowers. Most participants described the odor as "grandmalike" rather than as shoe leather odor. Notably, the odor memory scores were not affected by the poor identification of shoe leather, as all 4 target odors showed similar mean SSODMT scores. Apparently the false labeling of odors in the subject's mind prior to recognition testing did not affect testing performance. Other memorizing strategies might have been used unconsciously and, as suggested by the findings of Zelano et al, ${ }^{28}$ different brain areas can be used for the short-term memory involved in odor recognition, whether or not they are named.

The cognitive tests used here (ie, the MMSE, WST, and APM) were used to test cognitive function and to determine whether any of the participants had cognitive impairments that could influence their olfactory memory. The MMSE scores of all participants were within the normal range in terms of overall cognitive function. Regarding more specific cognitive functions, such as intelligence, the AMP scores correlated positively with the SSODMT scores, although this was analyzed only in a subgroup of 30 participants. The WST scores showed a weak negative correlation with the SSODMT scores and a positive correlation with age. This is most likely because the WST tests vocabulary or language abilities, which are influenced by the subject's educational level and linguistic experience (these presumably increase with age). Furthermore, the areas targeted by the AMP differ from those targeted by the WST. Indeed, the AMP has similarities to the odor memory test procedure and investigates nonverbal intelligence and logical abilities, which could affect odor memory performance.

In olfactory testing, normative data show a decline in olfactory function with increasing age. ${ }^{14} \mathrm{We}$ also found a decline in SSODMT and SSIT scores with increasing age. Therefore, larger study populations are needed to establish normative age-related data for the SSODMT. The SSODMT should also be tested in subjects with cognitive impairment to define cutoff scores that indicate odor memory malfunction.

Showing moderate to high correlation, the scores on the new SSODMT were higher in subjects with better olfactory function as measured by the SSIT. Previous studies found a similar relationship between olfactory function and odor memory, including results of the SSODMT in hyposmic patients. ${ }^{12,16,29}$ This highlights the need for people to have the ability to identify odors in order to develop odor memory as well as the need to test olfactory function in conjunction with memory tests. We found higher SSODMT scores when the experimental time period was longer (eg, 30 or 60 seconds rather than 10 seconds). Humans can show poor initial encoding and simultaneously have the ability to remember more odors over longer periods. ${ }^{25}$ The participants in our study may have needed more than 10 seconds to process odor information, which would explain why they showed worse odor memory after a 10 -second time period.

Subjective olfactory ratings did not correlate with olfactory test scores, including scores on the new SSODMT. Inconsistent subjective scoring relative to olfactory measurements is a common finding in routine clinical practice. ${ }^{30}$ Concerning differences in the ratings of female versus male participants, the women in our study showed significantly better self-assessment ratings than the men. This may be because of the unintended unbalanced distribution of women and men ( 30 women vs 18 men), or it may indicate that the women in this study had greater self-confidence in their olfactory function. These results again show the need for psychophysical tests in addition to questionnaires to get a complete view of an individual's olfactory and memory capacities.

Taken together, the results of this study demonstrate the usefulness of the SSODMT, a new odor discrimination memory test that uses Sniffin' Sticks, and they also show that it is comparable with the ODMT. The SSODMT is an easy-to-use and rapid way to routinely evaluate odor memory, and its cost is reasonable. Future studies are needed to establish normative data and to evaluate the use of the test in patients with diverse etiologies.

\section{Acknowledgments}

Leandra Jobs collected data for the present work in fulfillment of requirements for obtaining the degree Dr Med Univ.

\section{Declaration of Conflicting Interests}

The author(s) declared no potential conflicts of interest with respect to the research, authorship, and/or publication of this article.

\section{Funding}

The author(s) received no financial support for the research, authorship, and/or publication of this article.

\section{ORCID iD}

Gerold Besser (iD) https://orcid.org/0000-0003-3168-7477

\section{References}

1. Deems DA, Doty RL, Settle RG, et al. Smell and taste disorders, a study of 750 patients from the University of Pennsylvania Smell and Taste Center. Arch Otolaryngol Head Neck Surg. 1991;117(5):519-528. 
2. Doty RL, Deems DA, Stellar S. Olfactory dysfunction in parkinsonism: a general deficit unrelated to neurologic signs, disease stage, or disease duration. Neurology. 1988;38(8):1237-1244.

3. Haehner A, Boesveldt S, Berendse HW, et al. Prevalence of smell loss in Parkinson's disease - a multicenter study. Parkinsonism Relat Disord. 2009;15(7):490-494.

4. Doty RL. Olfaction in Parkinson's disease and related disorders. Neurobiol Dis. 2012;46(3):527-552.

5. Bahuleyan B, Singh S. Olfactory memory impairment in neurodegenerative diseases. JCDR. 2012;6(8):1437-1441.

6. Doty RL, Shaman P, Kimmelman CP, Dann MS. University of Pennsylvania Smell Identification Test: a rapid quantitative olfactory function test for the clinic. Laryngoscope. 1984; 94(2):176-178.

7. Kobal G, Klimek L, Wolfensberger M, et al. Multicenter investigation of 1,036 subjects using a standardized method for the assessment of olfactory function combining tests of odor identification, odor discrimination, and olfactory thresholds. Eur Arch Otorhinolaryngol. 2000;257(4):205-211.

8. Mueller C, Renner B. A new procedure for the short screening of olfactory function using five items from the "Sniffin' Sticks" identification test kit. Am J Rhinol. 2006;20(1): 113-116.

9. Mueller CA, Grassinger E, Naka A, Temmel AFP, Hummel T, Kobal G. A self-administered odor identification test procedure using the "Sniffin' Sticks." Chem Senses. 2006; 31(6):595-598.

10. Doty RL. Office procedures for quantitative assessment of olfactory function. Am J Rhinol. 2007;21(4):460-473.

11. Bromley SM, Doty RL. Odor recognition memory is better under bilateral than unilateral test conditions. Cortex. 1995; 31(1):25-40.

12. Choudhury ES, Moberg P, Doty RL. Influences of age and sex on a microencapsulated odor memory test. Chem Senses. 2003;28(9):799-805.

13. Hummel T, Sekinger B, Wolf SR, Pauli E, Kobal G. "Sniffin" Sticks': olfactory performance assessed by the combined testing of odor identification, odor discrimination and olfactory threshold. Chem Senses. 1997;22(1):39-52.

14. Hummel T, Kobal G, Gudziol H, Mackay-Sim A. Normative data for the "Sniffin' Sticks" including tests of odor identification, odor discrimination, and olfactory thresholds: an upgrade based on a group of more than 3,000 subjects. Eur Arch Otorhinolaryngol. 2007;264(3):237-243.
15. Zucco GM. Olfactory performance assessed via a new odor recognition test: reliability and normative data. Cogn Psychol. 2011;23(1):1-7.

16. Croy I, Zehner C, Larsson M, Zucco GM, Hummel T. Testretest reliability and validity of the Sniffin' TOM odor memory test. Chem Senses. 2015;40(3):173-179.

17. Chelonis JJ, Cox AR, Karr MJ, Prunty PK, Baldwin RL, Paule MG. Comparison of delayed matching-to-sample performance in monkeys and children. Behav Processes. 2014;103:261-268.

18. Folstein MF, Folstein SE, McHugh PR. "Mini-Mental State." Psychiatry Res. 1975;12(3):189-198.

19. Lehrl S, Triebig G, Fischer B. Multiple choice vocabulary test MWT as a valid and short test to estimate premorbid intelligence. Acta Neurol Scand. 1995;91(5):335-345.

20. Raven JC. APM Raven's Advanced Progressive Matrices. Berlin, Germany: Hogrefe Verlag; 2012.

21. Bors DA, Stokes TL. Raven's Advanced Progressive Matrices: norms for first-year university students and the development of a short form. Educ Psychol Meas. 1998;58:382-398.

22. Doty RL, McKeown DA, Lee WW, Shaman P. A study of the test-retest reliability of ten olfactory tests. Chem Senses. 1995;20(6):645-656.

23. Hummel T, Konnerth CG, Rosenheim K, Kobal G. Screening of olfactory function with a four-minute odor identification test: reliability, normative data, and investigations in patients with olfactory loss. Ann Otol Rhinol Laryngol. 2001;110(10):976-981.

24. Shepherd GM. Smell images and the flavour system in the human brain. Nature. 2006;444:316-321.

25. Doty RL. Handbook of Olfaction and Gustation. Hoboken, New Jersey: Wiley Blackwell; 2015.

26. McMahon C, Scadding GK. Le Nez du Vin - a quick test of olfaction. Clin Otolaryngol. 1996;21(3):278-280.

27. Cain WS. To know with the nose: keys to odor identification. Science. 1979;203(4379):467-470.

28. Zelano C, Montag J, Khan R, Sobel N. A specialized odor memory buffer in primary olfactory cortex. PLOS ONE. 2009;4(3):e4965.

29. Kollndorfer K, Reichert J, Braunsteiner J, Schöpf V. Assessment of olfactory memory in olfactory dysfunction. Perception. 2017;46(3-4):516-529.

30. Landis BN, Hummel T, Hugentobler M, Giger R, Lacroix JS. Ratings of overall olfactory function. Chem Senses. 2003; 28(8):691-694. 